Published in final edited form as:

Trends Biochem Sci. 2014 October ; 39(10): 447-456. doi:10.1016/j.tibs.2014.06.006.

\title{
The growth-defense pivot: Crisis management in plants mediated by LRR-RK surface receptors
}

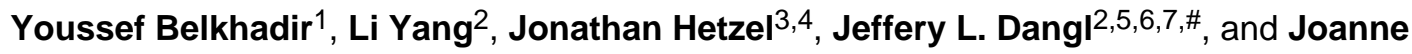 \\ Chory ${ }^{3,8, \#}$ \\ ${ }^{1}$ Gregor Mendel Institute (GMI), Austrian Academy of Sciences, Vienna Biocenter (VBC), Austria \\ ${ }^{2}$ Department of Biology and Howard Hughes Medical Institute, University of North Carolina at \\ Chapel Hill, Chapel Hill, NC 27599, USA \\ ${ }^{3}$ Plant Biology Laboratory, The Salk Institute for Biological Studies, La Jolla, CA USA, 92037 \\ ${ }^{4}$ Division of Biology, UCSD, Gilman Drive, La Jolla, CA 92037 \\ ${ }^{5}$ Microbiology and Immunology, Coker Hall \# 3280 University of North Carolina at Chapel Hill, \\ Chapel Hill, NC 27599, USA \\ ${ }^{6}$ Curriculum in Genetics, Coker Hall \# 3280 University of North Carolina at Chapel Hill, Chapel \\ Hill, NC 27599, USA \\ ${ }^{7}$ Carolina Center for Genome Sciences, Coker Hall \# 3280 University of North Carolina at Chapel \\ Hill, Chapel Hill, NC 27599, USA \\ ${ }^{8}$ Howard Hughes Medical Institute
}

\begin{abstract}
Plants must adapt to their environment and require mechanisms for sensing their surroundings and responding appropriately. An expanded family of greater than 200 leucine-rich repeat receptor kinases (LRR-RKs) transduces fluctuating and often contradictory signals from the environment into changes in nuclear gene expression. Two LRR-RKs, BRASSINOSTEROID INSENSITIVE 1 (BRI1), a steroid receptor, and FLAGELLIN-SENSITIVE 2 (FLS2), an innate immune receptor that recognizes bacterial flagellin, act cooperatively to partition necessary growth-defense tradeoffs. BRI1 and FLS2 share common signaling components and slightly different activation mechanisms. BRI1 and FLS2 are paradigms for understanding signaling mechanisms of LRRcontaining receptors in plants.
\end{abstract}

\section{Keywords}

Cell surface signaling; tradeoffs LRR-RKs; plant growth; innate immunity; crosstalk

\footnotetext{
(C) 2014 Elsevier Ltd. All rights reserved.

${ }^{\#}$ Co-corresponding authors: (chory@ salk.edu) or J.L.D. (dang1@email.unc.edu).
}

Publisher's Disclaimer: This is a PDF file of an unedited manuscript that has been accepted for publication. As a service to our customers we are providing this early version of the manuscript. The manuscript will undergo copyediting, typesetting, and review of the resulting proof before it is published in its final citable form. Please note that during the production process errors may be discovered which could affect the content, and all legal disclaimers that apply to the journal pertain. 


\section{Making sound decisions: To defend and grow}

Plants are stuck in an environment from which they cannot escape [1]. As such, discrimination of prolonged signals from a constant barrage of background noise, as well as proper integration of these inputs to endogenous growth programs, is fundamental to the success and survival of the 350,000 species of flowering plants that currently inhabit earth [1].

Plants process complex information from their local environment [2-5]. They can anticipate dawn and the seasons, remember winter in order to flower at the right time of year, and communicate with each other and with other species through unique, volatile metabolites made through sophisticated biochemical pathways [2, 5-7]. Because they are sessile, plants forage for light and nutrients through differential growth [5, 8]. Thus, plants invest carefully calibrated resources into their growth programs to satisfy their needs [9]. However, plants are constantly integrating signals from other living organisms that are on or very near their bodies, thus sorting pathogens and pests from mutualists and symbionts [10-15]. Because they cannot run away from attack, plants evolved a sophisticated two-tiered immune system that controls the production of a diverse arsenal of small molecules to fend off predators, defend themselves against pathogens, attract pollinators, and mediate communication within and between species $[13,14,16-18]$. Responding to living organisms like pathogens requires temporary modulation of investments made into growth [9]. When plants sense the presence of pathogens, they activate a series of energetically costly defense programs that eventually culminate in the cessation of pathogen proliferation $[9,17]$. Plants make regulatory decisions by processing information that originates at the single cell level and distributing secondary messengers locally and distally [17]. Thus, integration of environmental signals often requires management of growth-defense trade-offs [9].

Over the past two decades, many individual pathways that regulate growth and plant immune and defense responses have been elucidated [1, 3, 19, 20]. Yet we do not understand how this information is integrated within the plant either spatially or temporally to optimize the growth rate and body plan for life in a particular environment. This review is motivated by recent structural and biochemical studies that implicate a role for leucine-rich repeat receptor kinases (LRR-RKs) in the battle between growth and defense. We provide an overview of how the shared molecular components in these two distinct pathways act to finely balance growth-defense trade-offs in plants.

\section{Unique receptor kinases involved in growth, development and defense}

Plants and animals exploit extracellular leucine-rich repeats (eLRRs) for the perception of either self or non-self-derived signals at the cell surface [21, 22]. Forward genetic studies for mutations in plant growth, development, and defense converged on a role for LRR kinases in all of these processes [21, 23, 24]. More than 200 LRR-RKs have since been identified in the genome of Arabidopsis and these likely reflect an adaptive strategy in which plants use sophisticated extracellular sensors to determine the current and anticipated state of their growth environment (Figure 1) [25, 26]. 
LRR-RKs are typically characterized by a series of extracellular LRRs (eLRRs), a single transmembrane pass and, in most cases, a functional intracellular kinase domain. The kinase domains of LRR-RKs can be further divided into RD and non-RD families, based on the presence or absence of an arginine located prior to a catalytic aspartate residue [27]. NonRD kinases lack the strong autophosphorylation activities of RD kinases and display lower enzymatic activities [28]. The differences in activities possibly indicate that these two classes of kinases are subject to distinct regulatory mechanisms [27]. The eLRRs control the assembly of signaling competent LRR RK complexes by conferring ligand specificity and recruiting appropriate signaling partners [21, 22]. A comparison of the LRR-RK kinase domains shows a very close relationship between the proteins, which may support the model of a common protein kinase ancestor (Figure 1A) [26]. However, there are clear differences in the number of LRRs for each LRR-RK (Figure 1B). Analysis of LRR numbers demonstrates a bimodal distribution that separates the LRR-RKs into two groups with a median number of LRRs of 6 and 22, respectively (Figure 1B). Interestingly, proteins with closely related kinase domains also tend to have similar numbers of LRRs. For comparison, leucine-rich repeat receptor-like proteins (LRR-RLPs), which lack a kinase domain, do not show a bimodal distribution and instead have a higher average number of LRRs (Figure 1B). It appears that there is a functional connection between these two groups of LRR-RKs, representing receptors and co-receptors, respectively (discussed later). Importantly, LRRRLPs do not show this distribution because they might function differently, perhaps through direct ligand interaction.

\section{LRR-RKs are core modulators of plant growth}

Of the $\sim 8$ phytohormones that control plant growth, brassinosteroids (BRs), which are polyhydroxylated steroid hormones that play essential roles in nearly all phases of plant development, are the only class that utilizes a cell surface receptor [1, 3, 29-32]. Receptors for the other plant hormones are mainly distributed in the cytoplasm, nucleus or the endoplasmic reticulum (ER) $[1,3,33]$. Because of the striking and easy-to-score phenotypes of BR biosynthetic and response mutants (Figure 1C), the BR pathway is the best understood LRR-RK signal transduction pathway in plants [31, 34]. The organizational principles of BR signaling diverge radically from the paradigms of animal steroid signaling, which involve the members of a nuclear receptor superfamily (Box 1).

\section{Text Box}

\section{A few distinguishing organizational principles of plant steroid signaling pathways}

(1) Plants perceive steroid hormones at the cell surface using a membrane-bound LRRreceptor Ser/Thr/Tyr kinase, as opposed to the familiar nuclear receptors utilized by metazoans $[102,103]$. (2) The key signaling output is de-phosphorylation of the BES1/ BZR1 transcription factors, which homo-or hetero-dimerize to activate or repress target genes [48]. (3) With the exception of the receptor, BRI1, each BR signaling component is encoded by a gene family of 3 to 12 members, most with partially redundant functions (BIN2, 10-gene family [104]; BSU1, 4-gene family [47]; BESI/BZR1, 6 genes [105]; $B K I 1,7$ genes [40]; $B S K 1,12$ genes [42]; and $B A K 1,5$ genes [65]). Redundancy may be 
built into plant signaling systems to generate fine-tuned and cell-type specific responses. However, knowledge concerning the extent of the functional overlap between, and specific expression patterns of, gene family members is rather limited. (4) Response modulation by negative feedback mechanisms (through $B K I 1$ and repression of biosynthetic gene expression,) ensures that the response circuit is shut down when the initial signal drops below a threshold.

BRs are perceived by 3 LRR-kinases: BRI1, BRL1 and BRL3 (Figure 1A) [35]. BRI1 is ubiquitously expressed and accounts for most of the steroid binding activity [36, 37]. By contrast, BRL1 and BRL3 have very restricted expression in a subset of vascular and root cells where they subtly regulate shoot and root development [35, 36]. Loss-of-function mutations in either BR biosynthetic or signaling genes cause severe plant dwarfism, primarily due to a lack of cellular expansion [31,34].

Most BR signaling components are now known and a pathway linking steroid recognition at the cell surface to changes in gene expression has been proposed (Figure 2) [34, 38, 39]. Upon treatment of seedlings with brassinolide (BL), the most potent BR, BRI1 phosphorylates the negative regulator BRASSINOSTEROID KINASE INHIBITOR 1 (BKI1) on tyrosine 211 causing its displacement from the membrane to the cytosol where it is inactive [40]. The LRR-RK co-receptor BRI1-ASSOCIATED KINASE 1 (BAK1) can then freely associate with BRI1 to form a complex that elevates the signaling output of the pathway through a series of reciprocal transphosphorylation events [41]. In parallel, activated BRI1 phosphorylates the serine/threonine kinase BR SIGNALING KINASE 1 (BSK1) [42, 43]. BSK1 is a receptor-like cytoplasmic kinase (RLCK) that positively regulates the BR pathway by relaying BRI1 signals to downstream signaling components. Downstream from BSK1 is BRASSINOSTEROID INSENSITIVE 2 (BIN2), which was identified from a gain-of-function mutation (bin2-1D) that resulted in a dwarf mutant that is indistinguishable from bril loss-of-function mutants (Figure 1C) [44]. BIN2, a GSK3-type kinase, negatively regulates the pathway by phosphorylating the downstream transcription factors BRASSINAZOLE RESISTANT 1 (BZR1) and BRI1-EMS-suppressor 1 (BES1) on multiple sites (Figure 2A) [39, 45]. Upon treatment with BRs, BIN2 is dephosphorylated at tyrosine 200 by the protein phosphatase 1 (PP1)-like phosphatase BSU1, leading to its inactivation $[46,47]$. Further dephosphorylation of BES1 and BZR1 by protein phosphatase 2A (PP2A) allows them to multimerize as either homodimers or heterodimers on the promoters of target genes (Figure 2B) [48]. The current model for BR signaling suggests that the signal is amplified via a linear phosphorylation/dephosphorylation cascade leading to the transcriptional regulation of hundreds of genes and, ultimately, coordinated cell expansion $[49,50]$.

\section{LRR-RKs at the frontline of plant defenses}

LRR-RKs also play a critical role in plant immunity $[17,51]$. The plant response to invading pathogens involves the detection of microbe-associated molecular patterns (MAMPs) by high affinity pattern recognition receptors (PRRs) that survey the extracellular space [17, 51]. The detection of non-self molecules by PRRs drives a hierarchical and stereotypic 
defense response that ultimately results in the cessation of microbial growth and signaling to both nearby uninfected cells and systemic tissues $[17,51]$. Acute PRR signaling results in the accumulation of reactive oxygen intermediates (ROIs), ion channel activation, activation of defense-related mitogen activated protein kinases (MAPKs) and transcriptional reprogramming [17, 51]. Collectively, these responses constitute early MAMP-triggered immunity (MTI) and often occur over seconds, minutes and hours, respectively [17, 51]. Longer-term MTI responses, which occur over hours to days, include hormone biosynthesis, production of compounds with microcidal and/or microstatic activities and cell wall reinforcement through callose deposition [17, 51]. Unlike BRs, which act, from the receptor to specific transcription factors, through an essentially linear signaling pathway, MTI appears to act through a ramified signaling network, and the outputs noted above are often not linearly connected (Figures 2,3).

In plants, the bacterial flagellum is sensed by FLAGELLIN-SENSITIVE 2 (FLS2) [52]. Plants that are unable to sense a 22-amino acid epitope derived from bacterial flagella (flg22) are more susceptible to bacterial infection [53]. The molecular players acting with, or downstream of, FLS2 are emerging at a fast pace (Figure 3). However, FLS2 signaling is still under dissection (Figure 3). The recent discovery that NADPH oxidase respiratory burst oxidase homolog $\mathrm{D}$ (RbohD) physically interacts with FLS2 within minutes of flg22 treatment, driving a calcium increase and subsequent flg22-induced ROI production, represents a major milestone in closing this gap [54, 55]. Future studies, aimed at understanding how the FLS2 signaling complex is organized in light of specific phosphorylation events that drive signaling, will significantly advance our knowledge of the ramified structure of this complex signaling network.

\section{LRR-RK signaling pathways are core modulators of growth-defense tradeoffs}

Sustained MTI, achieved by non-physiological flg22 treatments, can result in a chronic response known as seedling growth inhibition (SGI) [56]. SGI has been interpreted to represent transient growth defense-tradeoffs occurring upon flg22 perception and can also be triggered by other MAMPs [57-59]. BAK1 acts as a co-receptor for both BRI1 and FLS2 (Figures 2, 3), and other PRRs [28, 56, 60-63]. In fact, recent studies demonstrated that the FLS2/BAK1 receptor pair remains functional when their respective cytosolic kinase domains are reversed by swapping, suggesting that receptor-co-receptor formation, acts simply as a molecular switch for receptor activation [64].

BAK1 is a positive regulator of both BR-mediated cell expansion and growth, and flg22triggered defenses. bak1 null mutants are incompletely insensitive to flg22 and BR, suggesting some redundancy at this level of the signaling hierarchy. The other members of the somatic embryogenesis receptor kinase family, of which BAK1 is the third member (SERK3), are obvious candidates for mediators of this redundancy [65]. Indeed, in addition to BAK1, BRI1 also associates with SERK1 and SERK4/BKK1 (BAK1-like 1) (Figure2) $[61,65]$. SERK1 and SERK4/BKK1 can, to some extent, compensate for the loss of BAK1 in BR signaling [65]. BAK1 and SERK4 also exhibit overlapping functions in MTI through their functional association with FLS2 (Figure 3) [62], though most of the activity is due to 
BAK1. BAK1/SERKs, therefore, seem to act as 'bilateral' regulators of cellular expansion and MTI.

\section{Atomic structures pave the way for a refined understanding of LRR-RK signaling}

The atomic structures of the extracellular domains (ECD) of the BRI1-BAK1, BRI1-SERK1 and FLS2-BAK1 receptor complexes were solved with their cognate ligands (Figure 4) [6670]. These spectacular structures help rationalize a decade of forward genetic and molecular studies, and will drive functional studies aimed at understanding the mechanisms underlying ligand-dependent LRR-RK activation. The plant eLRRs evolved different ligand-binding domains to recognize ligands, such as the plant steroid BL and the 22-amino acid flagellin peptide (Figure 4A) [67, 69, 70]. Although the extended flg22 peptide establishes many direct contacts along 14 LRRs in the concave side of the FLS2 solenoid, BL interacts with a loop-out island domain between LRRs 21-22 as well as LRRs 23-25 (Figure 4B). The overall shape and curvature of the plant-specific twisted horseshoe-like structures formed by BRI1 and FLS2 ECDs differ in only minor ways because of the rigidity of the LRR fold (Figure 4B) [67, 69, 70]. The important consensus emerging from these data is that the binding of flg22 or BL to the ECDs of FLS2 or BRI1, respectively, reveal distinct ligandinterfaces that are subsequently recognized by the LRRs of BAK1 (Figure 4C). Thus, the assembly of ternary LRR signaling-competent complexes (e.g. BRI1-BL-BAK1 or FLS2flg22-BAK1) seems to follow a 1:1:1 stoichiometry controlled by a specific sequence of limited local events driven by only minor structural rearrangements (Figure 4C) [67, 69, 70]. Understanding the adaptive value of BAK1 recruitment to several LRR-RK-ligand interfaces will be key to fully understand cell surface signaling in plants.

Although the BAK1/SERKs cannot display ligand-binding activities on their own, the structures establish BAK1 and SERK1 as "true" co-receptors that interact directly with both flg22 and BL (Figure 4C) [67, 69, 70]. Unlike the reported structures for various animal Toll-like receptor ectodomains, no homodimeric complexes were found in the crystallographic cell units of these plant eLRRs [71]. Thus, homo-oligomerisation may not be required for their activation. Nevertheless, some caution has to be taken when comparing structural data and proposed models based on in vivo data. For example, substituting a key residue involved in flg22 binding caused FLS2 to lose the ability to interact with flg22 and BAK1, but retain the ability to transduce flg22 signals in a transient protoplast overexpression reporter system [70]. Furthermore, substituting key residues that create the FLS2binding platform caused BAK1 to lose the ability to interact with FLS2 and to be phosphorylated upon flg22 treatment, but nevertheless retain flg22-driven activation of downstream MAPK signaling [70]. These discrepancies reveal that either the specific biochemical assays used do not faithfully reflect events occurring in wild-type cells, and/or that crystallographic knowledge is not sufficient to precisely understand the highly sophisticated activation mechanisms of LRR-RKs. Detailed in vivo functional studies at native transgenic expression levels in appropriate genetic backgrounds are necessary to fully validate the proposed crystallographic models. 
The structures essentially validate and reinforce the previous proposal of a "double-lock" mechanism whereby BRI1 and FLS2 activation were predicated upon the high affinity recruitment of the LRR domain of BAK1 to the respective ligand-bound LRRs [72]. The slight alteration in structure of the kinase domain of BRI1 in its inhibited and activated forms indicate, to some extent, that BL drives conformational juxtaposition of the intracellular kinase domains to favor transphosphorylation and subsequent activation of signaling [73].

\section{Is BAK1 a rate-limiting modulator of growth-defense trade-offs?}

In native conditions, detectable amounts of BAK1 are found in 'off-ligand' FLS2 andBRI1 immunoprecipitates (IPs), albeit at much lower levels than in the presence of the respective ligands $[62,72,74]$. Sustained BR signaling can inhibit FLS2-dependent responses in a unidirectional way, giving rise to the idea that BRI1 BAK1 complex formation during heightened BR signaling could make BAK1 limiting for FLS2 function [60, 75].

Measurement of FLS2 BAK1 complex formation in elevated BR signaling conditions showed no detectable decrease in FLS2-BAK1 association [75, 76], consistent with a model in which an optimal range of endogenous BR concentrations is required for balanced growth and MTI, presumably in a BAK1-independent fashion.

However, elevated BRI1 levels (OxBRI1; see Figure 1C) antagonize flg22-mediated signaling in a BAK1-dosage dependent manner, indicating that the co-receptor could be rate-limiting when MTI and BR signaling occur concurrently [60]. Because an increased interaction between BAK1 and FLS2 is still detectable upon flg22 treatment in OxBRI1 plants, elevated levels of BRI1 might interfere with BAK1-mediated phosphorylation of FLS2 [76]. Elevated BRI1 levels perhaps alter the competency of BAK1 for its activity in FLS2 signaling by 'phosphocoding' it in a manner that prevents appropriate BAK1dependent phosphorylation of FLS2. Support for this idea can be derived from recent work showing that the phosphorylation and release of BOTRYTIS-INDUCED KINASE1 (BIK1) from, and recruitment of BAK1 to, FLS2 after flg22 exposure is retained, even when a specific FLS2 phosphorylation site (Ser-938) required for full signaling is mutated [77]. Thus, proper association between BAK1 and FLS2, likely triggering a highly specific phosphocode, is probably needed to drive appropriate MTI signaling [28]. Future studies aimed at dissecting how the sequential phosphorylation and dissociation of BIK1 from its FLS2 complex is modulated by different doses of BRI1, BAK1, flg22 and BL will be instrumental to understand if BAK1-dependent and BAK1-independent mechanisms are used to regulate the BR-MTI nexus at the cell surface.

\section{Kinase-dead receptors keep LRR-RK signaling pathways in check}

The newly discovered BAK1-interacting RK2 (BIR2) negatively regulates BAK1-mediated flg22 responses (Figure 3) [78]. The structure of the kinase domain of BIR2 shows that it is an enzymatically dead kinase with an occluded nucleotide-binding site [79]. BIR2 interacts constitutively with BAK1, thereby preventing its interaction with FLS2 [78]. Flg22 signals lead to BAK1 dissociation from BIR2, enabling enhanced BAK1 recruitment to FLS2 [78]. BR-regulated responses are not affected in bir2 mutant plants, suggesting that BIR2 does not 
sequester BAK1 for amplified BR signaling in the presence of BL, or that this function is redundant (there are four closely related BIR proteins in the Arabidopsis reference genome [78]). However, single BL and flg22 treatments can independently trigger the release of BAK1 from BIR2 and this release is dramatically enhanced when plants are treated concomitantly with both ligands [78]. Thus, in addition to its regulatory role on MAMP signaling, BIR2 could compensate for the decreased availability of BAK1 when both BR and flg22 signaling need to occur in parallel. We envision that BIR2 acts as a "BAK1 reservoir" to modulate BAK1 distribution to at least FLS2 and BRI1. Future mechanistic studies need to dissect how BRs and flg22 regulate the release of BAK1 from BIR2.

\section{Common RLCKs act independently in distinct LRR-RKs signaling pathways}

Consistent with potential crosstalk between different LRR-RK signaling systems, the membrane-bound RLCKs BIK1 and BSK1 associate with both BRI1 and FLS2 in the absence of ligand (Figures 2, 3) [80-82]. BIK1 is differentially phosphorylated by BRI1 and FLS2 to de-repress downstream activation [80, 81]. Similarly BSK1, a key player in BR signaling as noted above, also associates with, and positively regulates, FLS2 signaling (Figures 2, 3) [43, 82]. Whether BIK1 and BSK1 dosage is important for the sustained function of FLS2 or BRI1 pathways remains an open question. Thus, although three direct physical intersection points linking BR and flg22 signaling have been identified (BAK1, BSK1 and BIK1), the precise mechanisms by which BR modulate MAMPs signaling at the receptor complex are not clear (Figure 5). The differential phosphorylation of the molecular players acting concomitantly with FLS2 and BRI1 could explain the inhibitory effects of BRs on MTI, but this supposition requires in-depth clarification [83]. The next challenge will then be to determine the structures of complete LRR-RK signaling complexes comprising the full-length receptors, ligands and downstream RLCKs. This ambitious goal will require the reconstitution of structurally and functionally intact receptors in a lipid bilayer for subsequent crystallography or cryo-EM analyses [84].

\section{LRR-RK initiated cross-regulation in the nucleus}

BR and MTI signaling pathways intersect downstream of the respective receptors (Figure 5). The demonstration that elevated BR signaling downstream of BIN2 suppresses MTI is a key achievement (Figure 5) [76]. BRs prioritize constitutive growth over induced MTI via BZR1, which elevates the expression of several WRKY transcription factors that negatively control early MTI outputs [76]. For this, BZR1 forms a complex with WRKY40, a known negative regulator of immune responses [76]. Thus, BZR1-WRKY40 appears to be an important regulatory decision-making node that integrates BR signals on MTI. Recently, two studies demonstrated that a bHLH protein, HBI1 (Homolog of brassinosteroid enhanced expression 2 interacting with IBH1), is a negative regulator of MTI (Figure 5) [85, 86]. HBII expression is enhanced by BR treatment and repressed by flg22-driven MTI; constitutive expression of HBI1 leads to reduced MTI [85, 86]. HBI1 is part of a tripartite regulatory module that also contains PACLOBUTRAZOL RESISTANT1 (PRE1) and ILI1BINDING bHLH PROTEIN1 (IBH1) [87]. PRE1, IBH1 and HBI1 form an antagonistic switch that controls BR-dependent transcriptional responses (Figure 5) [87]. In this switch, BZR1 can positively regulate HBI1 function through PRE1 expression and HBI1 possibly 
activates BZR1 indirectly, by promoting BR biosynthesis and signaling [87]. Thus, HBI1 acts in concert with BZR1 to balance BR-modulated growth and MTI output, through perhaps partly overlapping transcriptional networks.

\section{Concluding remarks}

The current paradigm, derived from analyses of BRI1- and FLS2-mediated signaling, suggests the involvement of further undiscovered LRR-RK signaling pathways regulating plant responses to their environment. Despite the clear interaction between MTI and BR signaling, the causal relevance of MTI modulation by endogenous BR concentrations over developmental time and in specific cell types is open to important investigations. We envision that LRR-RKs will involve complex and diverse ligand-recognition and activation mechanisms, perhaps more than initially anticipated. In this respect the structures of the LRR-RKs Clavata 1 (CLV1; Figure1A), SUPPRESSOR OF BIR1-1 (SOBIR1) and the PRR Elongation Factor Receptor (EFR) in complex with their ligands and co-receptors are eagerly awaited [58, 88-90]. EFR activation, which is also BAK1-dependent, is anticipated to be generic and should resemble that of FLS2 and BRI1 [28]. However CLV1 and SOBIR1 activation, which respectively require the LRR-RLPs Clavata 2 (CLV2), and Cladosporium fulvum 4 (Cf-4) for function will very likely uncover new perception and activation paradigms $[88,91]$. Additional discoveries are expected to further our understanding of the molecular mechanisms required to integrate the time-sensitive perception of environmentally derived signals against the backdrop of ongoing dynamic and coordinated developmental changes.

\section{Acknowledgments}

This work was supported by grants from the National Institutes of Health (5 R01GM94428 to J.C. and 1 RO1 GM107444 to J.L.D); the National Science Foundation (IOS-0649389 to J.C); the Gorodon and Betty Moore Foundation (to J.L.D.); the Austrian Academy of Science through the Gregor Mendel Institute (Y.B.); and the Howard Hughes Medical Institute (HHMI; J.L.D. and J.C.). L. Y. is funded in part by the Gordon and Betty Moore Foundation through Grant GBMF 2550.02 to the Life Sciences Research Foundation. J. H. is supported by the Cell and Molecular Genetics Training Grant to UCSD, and by gifts to the Salk Institute from the Rose Hill Foundation and the H.A. and Mary K. Chapman Charitable Trust. We also thank Yvon Jaillais for providing some figure templates and Cyril Zipfel for sharing data prior to publication.

\section{References}

1. Vert G, Chory J. Crosstalk in cellular signaling: background noise or the real thing? Developmental cell. 2011; 21:985-991. [PubMed: 22172668]

2. Chen M, Chory J. Phytochrome signaling mechanisms and the control of plant development. Trends in cell biology. 2011; 21:664-671. [PubMed: 21852137]

3. Jaillais Y, Chory J. Unraveling the paradoxes of plant hormone signaling integration. Nature structural \& molecular biology. 2010; 17:642-645.

4. Wigge PA. Ambient temperature signalling in plants. Current opinion in plant biology. 2013; 16:661-666. [PubMed: 24021869]

5. Song J, et al. Remembering the prolonged cold of winter. Current biology : CB. 2013; 23:R807811. [PubMed: 24028964]

6. Hersch M, et al. Light intensity modulates the regulatory network of the shade avoidance response in Arabidopsis. Proceedings of the National Academy of Sciences of the United States of America. 2014; 111:6515-6520. [PubMed: 24733935]

7. Baldwin IT. Plant volatiles. Current biology : CB. 2010; 20:R392-397. [PubMed: 20462477] 
8. Ciolfi A, et al. Dynamics of the shade-avoidance response in Arabidopsis. Plant physiology. 2013; 163:331-353. [PubMed: 23893169]

9. Meldau S, et al. Defence on demand: mechanisms behind optimal defence patterns. Annals of botany. 2012; 110:1503-1514. [PubMed: 23022676]

10. Lebeis SL, et al. Culturing a plant microbiome community at the cross-Rhodes. The New phytologist. 2012; 196:341-344. [PubMed: 22978611]

11. Peiffer JA, et al. Diversity and heritability of the maize rhizosphere microbiome under field conditions. Proceedings of the National Academy of Sciences of the United States of America. 2013; 110:6548-6553. [PubMed: 23576752]

12. Wagner MR, et al. Natural soil microbes alter flowering phenology and the intensity of selection on flowering time in a wild Arabidopsis relative. Ecology letters. 2014; 17:717-726. [PubMed: 24698177]

13. Berendsen RL, et al. The rhizosphere microbiome and plant health. Trends in plant science. 2012; 17:478-486. [PubMed: 22564542]

14. Pineda A, et al. Helping plants to deal with insects: the role of beneficial soil-borne microbes. Trends in plant science. 2010; 15:507-514. [PubMed: 20542720]

15. Zamioudis C, Pieterse CM. Modulation of host immunity by beneficial microbes. Molecular plantmicrobe interactions : MPMI. 2012; 25:139-150. [PubMed: 21995763]

16. Pangesti N, et al. Two-way plant mediated interactions between root-associated microbes and insects: from ecology to mechanisms. Frontiers in plant science. 2013; 4:414. [PubMed: 24167508]

17. Dangl JL, et al. Pivoting the plant immune system from dissection to deployment. Science (New York, NY ). 2013; 341:746-751.

18. Dicke M, Baldwin IT. The evolutionary context for herbivore-induced plant volatiles: beyond the 'cry for help'. Trends in plant science. 2010; 15:167-175. [PubMed: 20047849]

19. Pieterse CM, et al. Networking by small-molecule hormones in plant immunity. Nature chemical biology. 2009; 5:308-316.

20. Robert-Seilaniantz A, et al. Hormone crosstalk in plant disease and defense: more than just jasmonate-salicylate antagonism. Annual review of phytopathology. 2011; 49:317-343.

21. De Smet I, et al. Receptor-like kinases shape the plant. Nature cell biology. 2009; 11:1166-1173.

22. Song DH, Lee JO. Sensing of microbial molecular patterns by Toll-like receptors. Immunological reviews. 2012; 250:216-229. [PubMed: 23046132]

23. Torii KU. Leucine-rich repeat receptor kinases in plants: structure, function, and signal transduction pathways. International review of cytology. 2004; 234:1-46. [PubMed: 15066372]

24. Marshall A, et al. Tackling drought stress: receptor-like kinases present new approaches. The Plant cell. 2012; 24:2262-2278. [PubMed: 22693282]

25. Dievart A, et al. Leucine-rich repeat receptor kinases are sporadically distributed in eukaryotic genomes. BMC evolutionary biology. 2011; 11:367. [PubMed: 22185365]

26. Shiu SH, Bleecker AB. Plant receptor-like kinase gene family: diversity, function, and signaling. Science's STKE : signal transduction knowledge environment. 2001; 2001:re22.

27. Dardick C, et al. Non-arginine-aspartate (non-RD) kinases are associated with innate immune receptors that recognize conserved microbial signatures. Current opinion in plant biology. 2012; 15:358-366. [PubMed: 22658367]

28. Schwessinger B, et al. Phosphorylation-dependent differential regulation of plant growth, cell death, and innate immunity by the regulatory receptor-like kinase BAK1. PLoS genetics. 2011; 7:e1002046. [PubMed: 21593986]

29. Domagalska MA, Leyser O. Signal integration in the control of shoot branching. Nature reviews. Molecular cell biology. 2011; 12:211-221.

30. Stewart JL, Nemhauser JL. Do trees grow on money? Auxin as the currency of the cellular economy. Cold Spring Harbor perspectives in biology. 2010; 2:a001420. [PubMed: 20182619]

31. Belkhadir Y, Chory J. Brassinosteroid signaling: a paradigm for steroid hormone signaling from the cell surface. Science (New York, NY ). 2006; 314:1410-1411. 
32. Jaillais Y, Vert G. Brassinosteroids, gibberellins and light-mediated signalling are the three-way controls of plant sprouting. Nature cell biology. 2012; 14:788-790.

33. Qiao H, et al. Processing and subcellular trafficking of ER-tethered EIN2 control response to ethylene gas. Science (New York, NY ). 2012; 338:390-393.

34. Zhu JY, et al. Brassinosteroid signalling. Development (Cambridge, England). 2013; 140:16151620.

35. Fabregas N, et al. The brassinosteroid insensitive1-like3 signalosome complex regulates Arabidopsis root development. The Plant cell. 2013; 25:3377-3388. [PubMed: 24064770]

36. Cano-Delgado A, et al. BRL1 and BRL3 are novel brassinosteroid receptors that function in vascular differentiation in Arabidopsis. Development (Cambridge, England). 2004; 131:53415351.

37. She J, et al. Structural basis for differential recognition of brassinolide by its receptors. Protein \& cell. 2013; 4:475-482. [PubMed: 23709366]

38. Kim TW, Wang ZY. Brassinosteroid signal transduction from receptor kinases to transcription factors. Annual review of plant biology. 2010; 61:681-704.

39. Kim TW, et al. Brassinosteroid signal transduction from cell-surface receptor kinases to nuclear transcription factors. Nature cell biology. 2009; 11:1254-1260.

40. Jaillais $\mathrm{Y}$, et al. Tyrosine phosphorylation controls brassinosteroid receptor activation by triggering membrane release of its kinase inhibitor. Genes \& development. 2011; 25:232-237. [PubMed: 21289069]

41. Bajwa VS, et al. Identification and functional analysis of tomato BRI1 and BAK1 receptor kinase phosphorylation sites. Plant physiology. 2013; 163:30-42. [PubMed: 23843605]

42. Sreeramulu S, et al. BSKs are partially redundant positive regulators of brassinosteroid signaling in Arabidopsis. The Plant journal : for cell and molecular biology. 2013; 74:905-919. [PubMed: 23496207]

43. Tang W, et al. BSKs mediate signal transduction from the receptor kinase BRI1 in Arabidopsis. Science (New York, NY ). 2008; 321:557-560.

44. Li J, et al. BIN2, a new brassinosteroid-insensitive locus in Arabidopsis. Plant physiology. 2001; 127:14-22. [PubMed: 11553730]

45. Vert G, Chory J. Downstream nuclear events in brassinosteroid signalling. Nature. 2006; 441:96100. [PubMed: 16672972]

46. Kim TW, et al. The CDG1 kinase mediates brassinosteroid signal transduction from BRI1 receptor kinase to BSU1 phosphatase and GSK3-like kinase BIN2. Molecular cell. 2011; 43:561-571. [PubMed: 21855796]

47. Mora-Garcia S, et al. Nuclear protein phosphatases with Kelch-repeat domains modulate the response to brassinosteroids in Arabidopsis. Genes \& development. 2004; 18:448-460. [PubMed: 14977918]

48. Tang W, et al. PP2A activates brassinosteroid-responsive gene expression and plant growth by dephosphorylating BZR1. Nature cell biology. 2011; 13:124-131.

49. Yu X, et al. A brassinosteroid transcriptional network revealed by genome-wide identification of BESI target genes in Arabidopsis thaliana. The Plant journal : for cell and molecular biology. 2011; 65:634-646. [PubMed: 21214652]

50. Sun Y, et al. Integration of brassinosteroid signal transduction with the transcription network for plant growth regulation in Arabidopsis. Developmental cell. 2010; 19:765-777. [PubMed: 21074725]

51. Macho AP, Zipfel C. Plant PRRs and the Activation of Innate Immune Signaling. Molecular cell. 2014; 54:263-272. [PubMed: 24766890]

52. Gomez-Gomez L, Boller T. FLS2: an LRR receptor-like kinase involved in the perception of the bacterial elicitor flagellin in Arabidopsis. Molecular cell. 2000; 5:1003-1011. [PubMed: 10911994]

53. Zipfel C, et al. Bacterial disease resistance in Arabidopsis through flagellin perception. Nature. 2004; 428:764-767. [PubMed: 15085136] 
54. Kadota Y, et al. Direct regulation of the NADPH oxidase RBOHD by the PRR-associated kinase BIK1 during plant immunity. Molecular cell. 2014; 54:43-55. [PubMed: 24630626]

55. Li L, et al. The FLS2-associated kinase BIK1 directly phosphorylates the NADPH oxidase RbohD to control plant immunity. Cell host \& microbe. 2014; 15:329-338. [PubMed: 24629339]

56. Chinchilla D, et al. A flagellin-induced complex of the receptor FLS2 and BAK1 initiates plant defence. Nature. 2007; 448:497-500. [PubMed: 17625569]

57. Nekrasov V, et al. Control of the pattern-recognition receptor EFR by an ER protein complex in plant immunity. The EMBO journal. 2009; 28:3428-3438. [PubMed: 19763086]

58. Zipfel C, et al. Perception of the bacterial PAMP EF-Tu by the receptor EFR restricts Agrobacterium-mediated transformation. Cell. 2006; 125:749-760. [PubMed: 16713565]

59. Liu Z, et al. BIK1 interacts with PEPRs to mediate ethylene-induced immunity. Proceedings of the National Academy of Sciences of the United States of America. 2013; 110:6205-6210. [PubMed: 23431184]

60. Belkhadir Y, et al. Brassinosteroids modulate the efficiency of plant immune responses to microbeassociated molecular patterns. Proceedings of the National Academy of Sciences of the United States of America. 2012; 109:297-302. [PubMed: 22087001]

61. Albrecht C, et al. Arabidopsis SOMATIC EMBRYOGENESIS RECEPTOR KINASE proteins serve brassinosteroid-dependent and -independent signaling pathways. Plant physiology. 2008; 148:611-619. [PubMed: 18667726]

62. Roux M, et al. The Arabidopsis leucine-rich repeat receptor-like kinases BAK1/SERK3 and BKK1/SERK4 are required for innate immunity to hemibiotrophic and biotrophic pathogens. The Plant cell. 2011; 23:2440-2455. [PubMed: 21693696]

63. Lin W, et al. Tyrosine phosphorylation of protein kinase complex BAK1/BIK1 mediates Arabidopsis innate immunity. Proceedings of the National Academy of Sciences of the United States of America. 2014; 111:3632-3637. [PubMed: 24532660]

64. Albert M, et al. A two-hybrid-receptor assay demonstrates heteromer formation as switch-on for plant immune receptors. Plant physiology. 2013; 163:1504-1509. [PubMed: 24130196]

65. Gou X, et al. Genetic evidence for an indispensable role of somatic embryogenesis receptor kinases in brassinosteroid signaling. PLoS genetics. 2012; 8:e1002452. [PubMed: 22253607]

66. Hothorn M, et al. Structural basis of steroid hormone perception by the receptor kinase BRI1. Nature. 2011; 474:467-471. [PubMed: 21666665]

67. Santiago J, et al. Molecular mechanism for plant steroid receptor activation by somatic embryogenesis co-receptor kinases. Science (New York, NY ). 2013; 341:889-892.

68. She J, et al. Structural insight into brassinosteroid perception by BRI1. Nature. 2011; 474:472-476. [PubMed: 21666666]

69. Sun Y, et al. Structure reveals that BAK1 as a co-receptor recognizes the BRI1-bound brassinolide. Cell research. 2013; 23:1326-1329. [PubMed: 24126715]

70. Sun Y, et al. Structural basis for flg22-induced activation of the Arabidopsis FLS2-BAK1 immune complex. Science (New York, NY ). 2013; 342:624-628.

71. Botos I, et al. The structural biology of Toll-like receptors. Structure (London, England : 1993). 2011; 19:447-459.

72. Jaillais Y, et al. Extracellular leucine-rich repeats as a platform for receptor/coreceptor complex formation. Proceedings of the National Academy of Sciences of the United States of America. 2011; 108:8503-8507. [PubMed: 21464298]

73. Bojar D, et al. Crystal structures of the phosphorylated BRI1 kinase domain and implications for brassinosteroid signal initiation. The Plant journal : for cell and molecular biology. 2014; 78:3143. [PubMed: 24461462]

74. Bucherl CA, et al. Visualization of BRI1 and BAK1(SERK3) membrane receptor heterooligomers during brassinosteroid signaling. Plant physiology. 2013; 162:1911-1925. [PubMed: 23796795]

75. Albrecht $\mathrm{C}$, et al. Brassinosteroids inhibit pathogen-associated molecular pattern-triggered immune signaling independent of the receptor kinase BAK1. Proceedings of the National Academy of Sciences of the United States of America. 2012; 109:303-308. [PubMed: 22087006] 
76. Lozano-Duran R, et al. The transcriptional regulator BZR1 mediates trade-off between plant innate immunity and growth. eLife. 2013; 2:e00983. [PubMed: 24381244]

77. Cao Y, et al. Mutations in FLS2 Ser-938 dissect signaling activation in FLS2-mediated Arabidopsis immunity. PLoS pathogens. 2013; 9:e1003313. [PubMed: 23637603]

78. Halter T, et al. The leucine-rich repeat receptor kinase BIR2 is a negative regulator of BAK1 in plant immunity. Current biology : CB. 2014; 24:134-143. [PubMed: 24388849]

79. Blaum BS, et al. Structure of the pseudokinase domain of BIR2, a regulator of BAK1-mediated immune signaling in Arabidopsis. Journal of structural biology. 2014; 186:112-121. [PubMed: 24556575]

80. Lin W, et al. Inverse modulation of plant immune and brassinosteroid signaling pathways by the receptor-like cytoplasmic kinase BIK1. Proceedings of the National Academy of Sciences of the United States of America. 2013; 110:12114-12119. [PubMed: 23818580]

81. Lu D, et al. A receptor-like cytoplasmic kinase, BIK1, associates with a flagellin receptor complex to initiate plant innate immunity. Proceedings of the National Academy of Sciences of the United States of America. 2010; 107:496-501. [PubMed: 20018686]

82. Shi H, et al. BR-SIGNALING KINASE1 physically associates with FLAGELLIN SENSING2 and regulates plant innate immunity in Arabidopsis. The Plant cell. 2013; 25:1143-1157. [PubMed: 23532072]

83. $\mathrm{Xu}$ J, et al. Identification and functional analysis of phosphorylation residues of the Arabidopsis BOTRYTIS-INDUCED KINASE1. Protein \& cell. 2013; 4:771-781. [PubMed: 24104392]

84. Song F, et al. Cryo-EM study of the chromatin fiber reveals a double helix twisted by tetranucleosomal units. Science (New York, NY ). 2014; 344:376-380.

85. Fan M, et al. The bHLH transcription factor HBI1 mediates the trade-off between growth and pathogen-associated molecular pattern-triggered immunity in Arabidopsis. The Plant cell. 2014; 26:828-841. [PubMed: 24550223]

86. Malinovsky FG, et al. Antagonistic regulation of growth and immunity by the Arabidopsis basic helix-loop-helix transcription factor homolog of brassinosteroid enhanced expression 2 interacting with increased leaf inclination1 binding bHLH1. Plant physiology. 2014; 164:1443-1455. [PubMed: 24443525]

87. Bai MY, et al. A triple helix-loop-helix/basic helix-loop-helix cascade controls cell elongation downstream of multiple hormonal and environmental signaling pathways in Arabidopsis. The Plant cell. 2012; 24:4917-4929. [PubMed: 23221598]

88. Nimchuk ZL, et al. Plant stem cell signaling involves ligand-dependent trafficking of the CLAVATA1 receptor kinase. Current biology : CB. 2011; 21:345-352. [PubMed: 21333538]

89. Liebrand TW, et al. Receptor-like kinase SOBIR1/EVR interacts with receptor-like proteins in plant immunity against fungal infection. Proceedings of the National Academy of Sciences of the United States of America. 2013; 110:10010-10015. [PubMed: 23716655]

90. Zhang W, et al. Arabidopsis receptor-like protein30 and receptor-like kinase suppressor of BIR1-1/ EVERSHED mediate innate immunity to necrotrophic fungi. The Plant cell. 2013; 25:4227-4241. [PubMed: 24104566]

91. Liebrand TW, et al. Two for all: receptor-associated kinases SOBIR1 and BAK1. Trends in plant science. 2014; 19:123-132. [PubMed: 24238702]

92. Sonnhammer EL, et al. Pfam: a comprehensive database of protein domain families based on seed alignments. Proteins. 1997; 28:405-420. [PubMed: 9223186]

93. Kumar S, et al. MEGA2: molecular evolutionary genetics analysis software. Bioinformatics. 2001; 17:1244-1245. [PubMed: 11751241]

94. Oh MH, et al. The Carboxy-terminus of BAK1 regulates kinase activity and is required for normal growth of Arabidopsis. Frontiers in plant science. 2014; 5:16. [PubMed: 24550926]

95. Gampala SS, et al. An essential role for 14-3-3 proteins in brassinosteroid signal transduction in Arabidopsis. Developmental cell. 2007; 13:177-189. [PubMed: 17681130]

96. Wang H, et al. Dual role of BKI1 and 14-3-3 s in brassinosteroid signaling to link receptor with transcription factors. Developmental cell. 2011; 21:825-834. [PubMed: 22075146]

97. Wu G, et al. Methylation of a phosphatase specifies dephosphorylation and degradation of activated brassinosteroid receptors. Science signaling. 2011; 4:ra29. [PubMed: 21558554] 
98. Irani NG, et al. Fluorescent castasterone reveals BRI1 signaling from the plasma membrane. Nature chemical biology. 2012; 8:583-589.

99. Wang X, et al. Autoregulation and homodimerization are involved in the activation of the plant steroid receptor BRI1. Developmental cell. 2005; 8:855-865. [PubMed: 15935775]

100. Robatzek S, Wirthmueller L. Mapping FLS2 function to structure: LRRs, kinase and its working bits. Protoplasma. 2013; 250:671-681. [PubMed: 23053766]

101. Beck M, et al. Spatio-temporal cellular dynamics of the Arabidopsis flagellin receptor reveal activation status-dependent endosomal sorting. The Plant cell. 2012; 24:4205-4219. [PubMed: 23085733]

102. Margolis RN, Christakos S. The nuclear receptor superfamily of steroid hormones and vitamin D gene regulation. An update. Annals of the New York Academy of Sciences. 2010; 1192:208214. [PubMed: 20392238]

103. Thummel CS, Chory J. Steroid signaling in plants and insects--common themes, different pathways. Genes \& development. 2002; 16:3113-3129. [PubMed: 12502734]

104. Galvan-Ampudia CS, Vernoux T. Signal integration by GSK3 kinases in the root. Nature cell biology. 2014; 16:21-23.

105. Guo H, et al. Mechanisms and networks for brassinosteroid regulated gene expression. Current opinion in plant biology. 2013; 16:545-553. [PubMed: 23993372] 


\section{Highlights}

LRR-RKs are the largest receptor class in plants.

Plant LRR-RKs have a unique activation mechanism.

LRR-RKs are dual specificity kinases.

LRR-RKs signal through common and unique signaling components. 

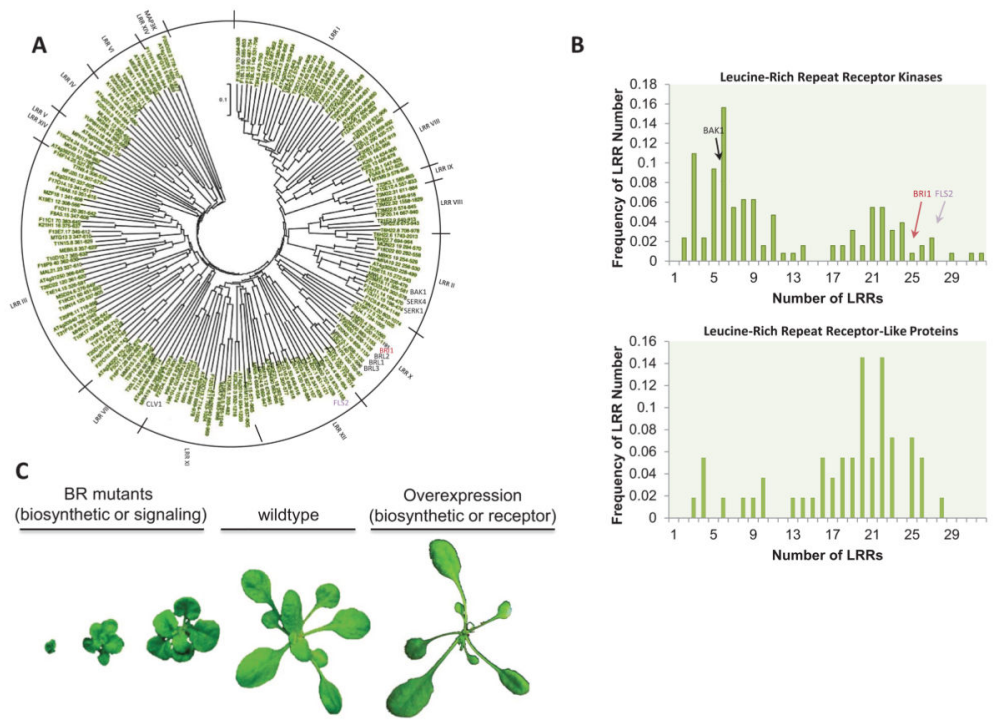

Figure 1. A comparison of LRR-RK kinase domains and LRR numbers

A- The kinase domains of LRR-RKs in the Arabidopsis genome were aligned by HMMER [92], a neighbor-joining phylogenetic tree was created for the kinase domains alignment using MEGA [93], and the phylogenetic tree was tested with bootstrapping. LRR-RKs discussed in the review are labeled accordingly. The kinase subfamilies are indicated along the outer edge of the alignment.

B- LRRs for each LRR-RK from the kinase alignment were identified using HMMER for domain analysis with a low stringency for the LRR domain from the PFAM database PF00560 [92]. For comparison the LRRs from LRR-RLPs were identified using the PFAM database as well. The total number of LRRs for each LRR-RK and LRR-RLP were calculated to give the distribution of number of LRRs seen in these proteins. The frequency of each number of LRRs was determined by dividing each value by the total number of LRR-RKs and LRR-RLPs analyzed and then plotting the result. The validity of this method was verified using known LRR numbers based on crystal structures and analyzing several LRR-RKs by hand. LRR-RKs discussed in the review are labeled accordingly.

C- Mutations in pathways that are controlled by LRR-RKs can have obvious growth and development phenotypes. Disruption of endogenous BR signaling through loss-of-function mutations in steroid biosynthesis or signaling translates into severe dwarfism. Opposite effects can be achieved by gain-of-function mutations that elevate signaling through increased biosynthesis or receptor overexpression.

Abbreviations: LRR-RKs, leucine-rich repeat receptor kinases; LRR-RLPs, leucine-rich repeat receptor-like proteins; BR, brassinosteroid. 

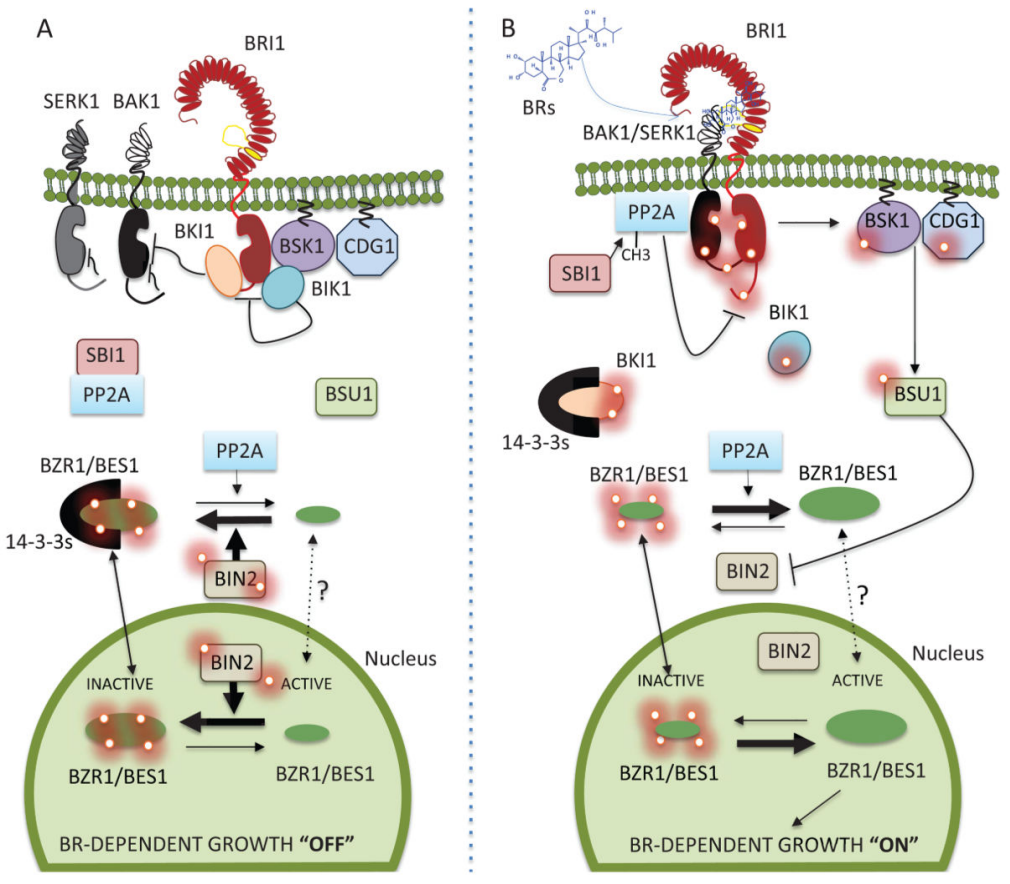

Figure 2. A model of BR signaling from the cell surface to the nucleus

A- In the absence of BR, signal-competent BRI1 is regulated by "cis" and "trans" mechanisms. The kinase domains of BAK1 and BRI1 are both inhibited by their own Cterminal tail [94]. BRI1 activity is further inhibited by an association with BKI1, a plasma membrane-associated phosphoprotein. In this configuration, BIN2, exists in an active and phosphorylated form. As a negative regulator of BR signaling, cytoplasmic and nuclear localized BIN2 inactivates BZR1 and BES1, two plant specific transcription factors. BIN2 has a dual action on BZR1/BES1: First, by phosphorylating them in the cytoplasm, BIN2 promotes their cytoplasmic retention by phosphopeptide-binding 14-3-3 proteins [95]; second, by phosphorylating them in the nucleus BIN2 blocks their DNA binding and transcriptional activities.

B- Binding of BR to BRI1 triggers the Tyrosine phosphorylation of BKI1 and its subsequent dissociation from the plasma membrane, thereby allowing the recruitment of BAK1/SERK3 or SERK1. Meanwhile, released, cytosolic BKI1 interacts with and thus titrates 14-3-3 proteins away from BZR1 $[95,96]$, promoting the accumulation of BZR1/BES1 in the nucleus in their active forms. In the absence of ligand, BAK1/SERK1 are auto-inhibited by their C-terminal tails; this inhibition is relieved by binding to BRI1 [94]. After a series of reciprocal trans-phosphorylation events on Ser-Thr and Tyr residues, signal competent BRI1-BAK1 hetero-oligomers phosphorylate the RLCKs BSK1 and CDG1, which in turn activate BSU1; BSU1 dephosphorylates and inactivates BIN2. The inactivation of BIN2, coupled with the activities of a PP2A, allow for the accumulation of BZR1/BES1 in their dephosphorylated active forms. BZR1/BES1 can multimerize either on their own or together with other transcription factors to bind target promoters to either repress or activate the expression of hundreds of genes to optimize BR-regulated growth (see Figure 5). Once BR signals have been transduced, the leucine carboxylmethyltransferase SBI1 promotes PP2A association with the plasma membrane by methylating it [97]. Once at the membrane, PP2A 
triggers specific termination of BR signaling via BRI1 dephosphorylation and subsequent degradation [97].

The BL docking platform is depicted in yellow. BRI1 constitutively cycles between the plasma membrane and early endosomal compartments and can signal from either location (not shown here, [98]). BRI1 may also exist as pre-formed homo-oligomers at the cell surface [99]. For simplicity, we show BRI1 as a monomer. Phosphorylation events are indicated by the small white circles surrounded by a fluorescent red halo. The question mark highlights knowledge gaps in the signaling pathway.

Abbreviations: BR, brassinosteroid; BRI1, BRASSINOSTEROID INSENSITIVE 1; BAK1, BRI1-ASSOCIATED KINASE 1; BKI1, BRI1 KINASE INHIBITOR 1; BSK1; BIN2, BRASSINOSTEROID INSENSITIVE 2; BZR1, BRASSINAZOLE RESISTANT 1; BES1, BRI1-EMS-suppressor 1; RLCK, receptor like cytoplasmic kinase; CDG1, Constitutive Differential Growth 1; SBI1, SUPPRESSOR OF bri1; BSU1; bri1 SUPPRESSOR 1; PP2A, PROTEIN PHOSPHATASE 2A. 

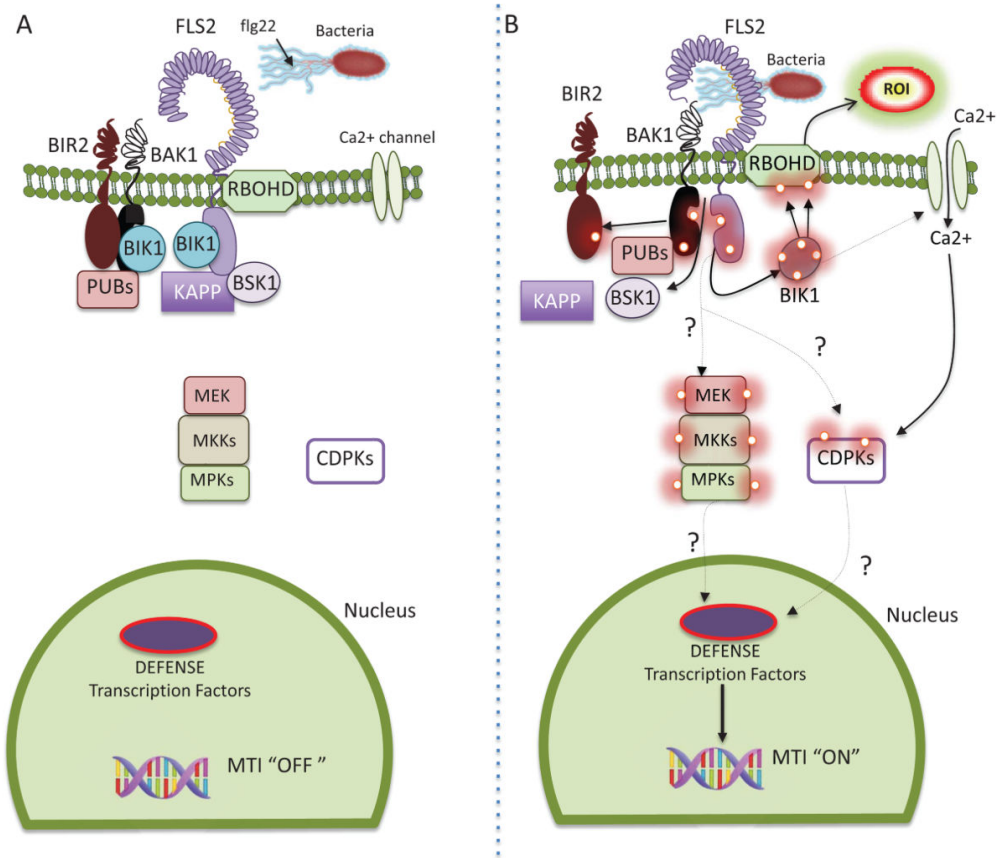

Figure 3. A model of FLS2-controlled defense in Arabidopsis

A- In the absence of ligand, the signaling potential of FLS2 is kept in check by its association with KAPP [100]. In this signal-competent state, FLS2 associates with at least BIK1, BSK1 and RbohD. BIK1 is a member of the family of AvrPphB SUSCEPTIBLE1 (PBS1)-like proteins (PBLs). Inactive FLS2 cycles on and off the plasma membrane via Brefeldin A-sensitive vesicle transport [101]. PBLs belong to a small subfamily of acylated RLCKs. BAK1 also associates with BIK1 in the absence of flg22 and is sequestered away from FLS2 by BIR2. BSK1 (see Figure 2) also associates with FLS2 to positively regulate flg22-dependent signaling.

B- As in BR signaling, flg22 detection triggers the very rapid formation of an FLS2-BAK1 hetero-oligomer. Flg22 binding allows the release of BAK1 from BIR2 and the dissociation or inactivation of KAPP phosphatases. The FLS2-BAK1 oligomer undergoes a series of trans-phosphorylation events and phosphorylates BIK1 at both tyrosine and serine/threonine residues. BIK1 auto-phosphorylates on multiple Tyr residues, trans-phosphorylates FLS2 and BAK1, and then dissociates from the complex. BIK1 targets at least the NADPH oxidase RBOHD for phosphorylation, activating an extracellular superoxide burst and intracellular calcium increase. Downstream of receptor activation, the MAP kinases MEKs, MKKs and MPKs and CDPKs are activated by an unknown mechanism and may contribute to induction of flg22-responsive genes. Once flg22 signals have been transduced, BSK1 partly dissociates from the receptor complex and BAK1 phosphorylates closely related PUBs, which in turn ubiquitylate FLS2 to regulate its abundance at the cell surface [100]. The flg22-docking platform is depicted in yellow in the concave side of FLS2 LRRs. Much as BRI1, FLS2 can exist as a homo-oligomer. For clarity we depict FLS2 as a monomer. Phosphorylation events are indicated by the small white circles surrounded by a fluorescent red halo. The question mark associated with dashed arrows highlights important knowledge gaps in the signaling pathway. 
Abbreviations: FLS2, FLAGELLIN SENSITIVE 2, KAPP, kinase-associated protein phosphatase; BIK1, BOTRYTIS INDUCED KINASE 1; BSK1; BR SIGNALING KINASE 1; RbohD, Respiratory burst oxidase homolog D; PBS1, AvrPphB SUSCEPTIBLE1; PBLs; (PBS1)-like protein; RLCKs, receptor like cytoplasmic kinases; BAK1, BRI1-

ASSOCIATED KINASE 1; flg22, flagellin 22; BIR2, BAK1-interacting Receptor kinase 2; MAPK/MPKs, mitogen activated protein kinases; MEK/MKKs, mitogen activated protein kinase kinase; CDPKs, calcium-dependent protein kinases; PUBs, U-Box E3 ubiquitin ligase proteins. 


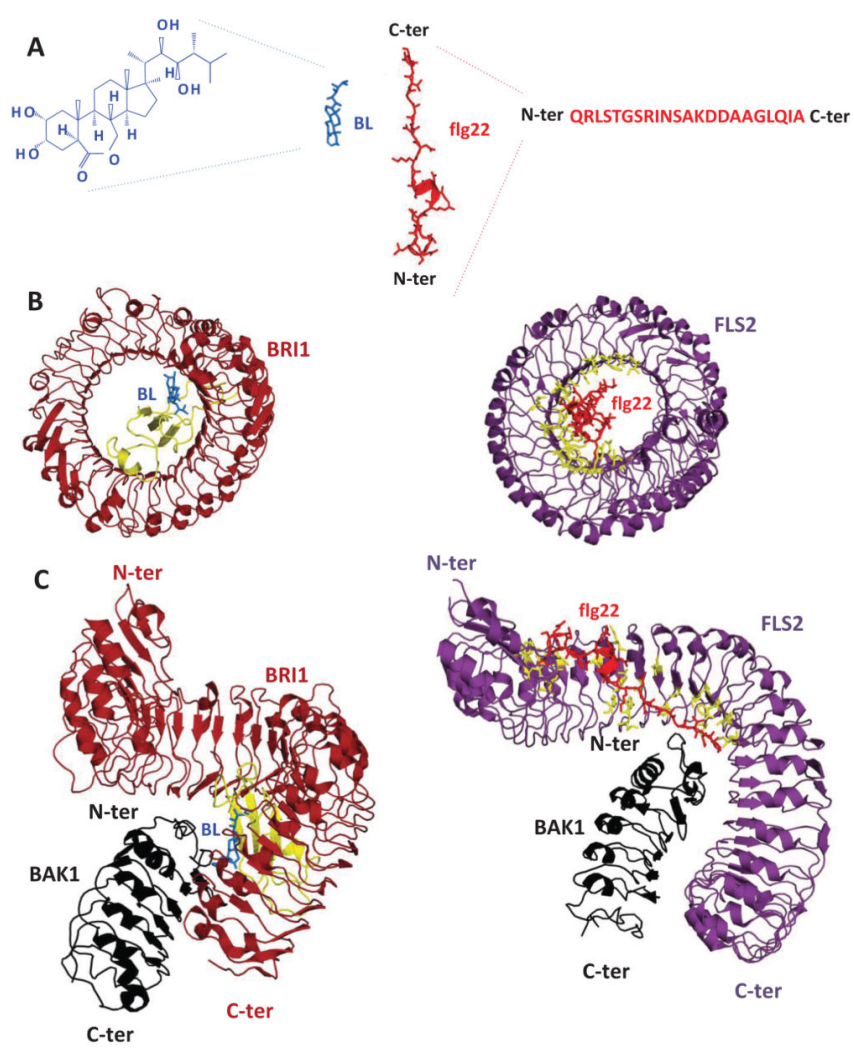

Figure 4. Atomic structures of ligands, receptor and co-receptor LRR complexes A- Comparison of BL (in blue) and flg22 (in red) atomic structures to scale.

B- Top-down of BRI1 (in red) and FLS2 (in purple) ECDs in complex with their respective ligands. BL (in blue) interacts with a 70 amino-acid loop-out island domain (in yellow) between LRRs20-21 and lays on the surface of LRRs 21-25 (of 25 total LRRs). The flg22 (in red) binding platform is distributed across 14 LRRs (in yellow) of FLS2 (28 LRRs total). Flg22 establishes direct links with amino-acids that project their lateral chains from the concave side of the FLS2 LRR solenoid (LRR3-16; in yellow). The central part of flg22 interacts with the lateral chains of residues derived from FLS2 LRR2-6. FLS2 specifically recognizes the $\mathrm{C}$ - and $\mathrm{N}$-terminal segment of flg22.

C- The N-terminal capping domain of BAK1 (in black) is important for recognition of BL (in blue) and folds on top of the BRI1 steroid binding pocket (in yellow) where it establishes contacts with the BRI1 LRR 25 and with the hormone itself. Bulky amino acids distributed throughout the BAK1 ECD also establish an interaction with the BRI1 C-terminal capping domain. In complex with FLS2, the $\mathrm{N}$ terminus of BAK1 associates through two separate interfaces involving LRRs 18-20 and 23-26 of FLS2. Mutations of key amino acids in either of them greatly reduce the binding of BAK1 to FLS2.

Abbreviations: BL, brassinolide; flg22, flagellin 22; BRI1, BRASSINOSTEROID INSENSITIVE 1; FLS2, FLAGELLIN SENSITIVE 2; ECDs, extracellular domains; LRRs, leucine-rich repeat; BAK1, BRI1-ASSOCIATED KINASE 1. 


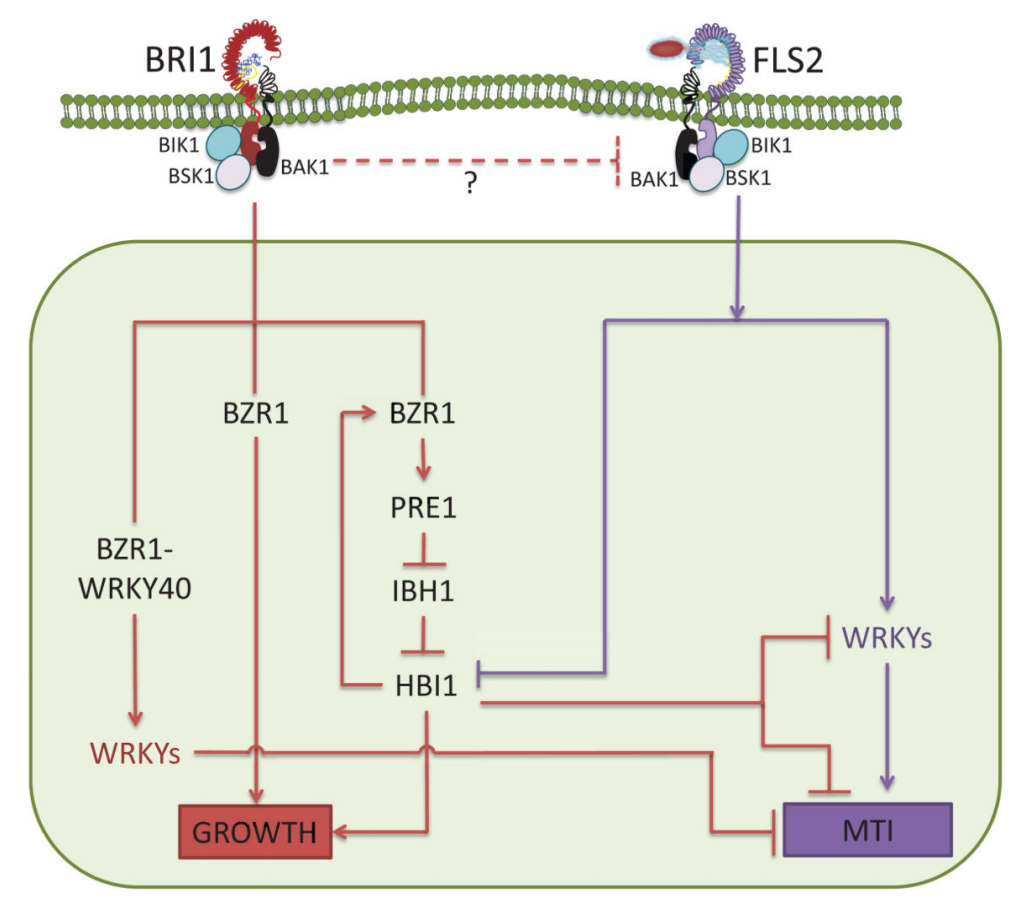

Figure 5. Network representation of BR and flg22 signaling crosstalk in the nucleus Transcriptional regulation is the final level of complexity in the growth-defense nexus between BR signaling and MTI. BZR1 integrates BR signals to repress MTI. BZR1 forms a complex with WRKY40 to up-regulate the expression of additional WRKY transcription factors that suppress MTI. HBI1 is another transcription factor performing dual roles in promoting growth and suppressing defense. HBI1 activates an overlapping set of positive regulators of cell elongation with BZR1, and represses the expression of defense genes including some WRKYs. BZR1 and HBI1 form a positive feedback loop in which BZR1 activates PRE1 expression to block IBH1-mediated inhibition of HBI1; HBI1 subsequently activates BZR1 by promoting BR biosynthesis and signaling. However, the activation of FLS2-mediated MTI inhibits the transcription of HBI1, demonstrating an antagonistic crosstalk between BR signaling and MTI at the HBI1 node. Signaling events downstream of BRI1 and FLS2 are annotated in red and purple, respectively. The rounded green rectangle represents a nucleus.

Abbreviations: BR, brassinosteroid; MTI, Microbes associated molecular patterns Trigerred Immunity, BZR1, BRASSINAZOLE RESISTANT 1 ; WRKY40 W-box binding transcritpion factor 40; HBI1, HOMOLOG OF BRASSINOSTEROID ENHANCED EXPRESSION 2 INTERACTING WITH IBH1; IBH1, ILI1-BINDING bHLH PROTEIN1 PRE1, PACLOBUTRAZOL RESISTANT1. 